\title{
Complex case of Takotsubo cardiomyopathy
}

\author{
Cătălina Arsenescu-Georgescu ${ }^{1,2}$, Mircea Balasanian ${ }^{1,2}$, Nicușor Lovin ${ }^{1}$, Larisa \\ Anghel* ${ }^{* 1,2}$
}

1"Prof. Dr. George I. M. Georgescu" Institute of Cardiovascular Diseases lasi, Romania, ${ }^{2 "}$ Grigore T. Popa" University of Medicine and Pharmacy, lasi, Romania

\begin{abstract}
Takotsubo cardiomyopathy, also known as stress-induced cardiomyopathy, is an unusual form of acute cardiomyopathy triggered by intense physical or emotional positive and negative stress. It involves left ventricular apical ballooning and mimics acute myocardial infarction. We present the case of a 74 year-old woman addressed to our clinic with the hypothesis of acute coronary syndrome in the context of $T$ wave inversions in anterior leads associated with atypical chest pain and acute pain in the left lower limb. She reported medical history of hypertension and repeated episodes of deep vein thrombosis with therapeutic limitations to anticoagulation and antiplatelet therapy because of the side effects. Due to high suspicion of acute coronary syndrome she underwent emergency coronary angiography, which revealed no significant coronary artery lesions but cardiac catheterization showed severe anterior-apical akinesia with compensatory infero-posterior hyperkinesis. This case report represents a diagnostic and also a therapeutic challenge because of the fortuitous diagnosis in the context of acute pain in the left lower limb which may cause confusion with the diagnosis of deep vein thrombosis. The acute pain in the left lower limb may also be considered the trigger factor for the Takotsubo cardiomyopathy in our patient.

Keywords: Takotsubo cardiomyopathy, coronary angiography, ventriculography
\end{abstract}

\section{Introduction}

Takotsubo cardiomyopathy (TCM), also known as, transient left ventricular apical ballooning syndrome" and "broke heart syndrome", is an acute cardiac condition that involves left ventricular apical ballooning and mimics acute myocardial infarction. His name comes from the Japanese, "tako tsubo" which translates to "octopus pot", a fishing jar with a narrow neck and wide base used to trap octopus, and describes the visual appearance of the heart on left ventriculography [1].

This cardiomyopathy occurs primarily in women in the seventh and eighth decades of life and is often associated with an acute physiological or emotional positive and negative stress. Takotsubo cardiomyopathy

Received: March 2017; Accepted after review: August 2017; Published: September 2017.

${ }^{*}$ Corresponding author: Larisa Anghel, Institute of Cardiovascular Diseases "Prof. Dr. George I. M. Georgescu”, 50 Carol I Blv, lasi, Romania.

E-mail: larisamed86@yahoo.com. has increasingly been reported in the medical literature in recent years [2-4]. It is important to consider this diagnosis, as patients may present to the emergency department with what appears to be uncomplicated acute coronary syndrome. They may present with symptoms of chest pain, ST-segment elevation and cardiac biomarkers consistent with an acute coronary syndrome. However, the echocardiography founds the ballooning of the left ventricular apex, which usually resolves in some weeks and the angiography finds no significant coronary stenosis. Much is still unknown regarding risk factors and clinical relationships but it seems to be triggered by emotional or physical stress [5].

\section{Case report}

A 74 year-old Caucasian female presented with chest pain for three days associated with acute pain in the left lower limb. The pain was described as 5/10 nonradiating, substernal, 
with associated asthenia. Vital signs were: Blood pressure of $120 / 70 \mathrm{~mm} \mathrm{Hg}$, pulse of 70 beats $/ \mathrm{min}$, respirations of 24 breaths $/ \mathrm{min}, \mathrm{O}_{2}$ saturation of $96 \%$ on room air, and temperature of $36^{\circ} \mathrm{C}$. Past medical history included hypertension and repeated episodes of deep vein thrombosis with therapeutic limitations to anticoagulation and antiplatelet therapy due to side effects. Also, ten years ago the patient was diagnosed with left breast cancer and underwent surgery. Her cardiac exam was unremarkable and she had important pain in the left lower limb but without clinical signs of deep vein thrombosis.

Initial ECG revealed normal sinus rhythm with a rate of $60 / \mathrm{min}$ and $T$ wave inversions in anterior leads (Figure 1). Chest radiography revealed no acute disease. Lab studies showed troponin I of $0.264 \mathrm{ng} / \mathrm{ml}$ and normal white blood, serum electrolytes and coagulation studies.

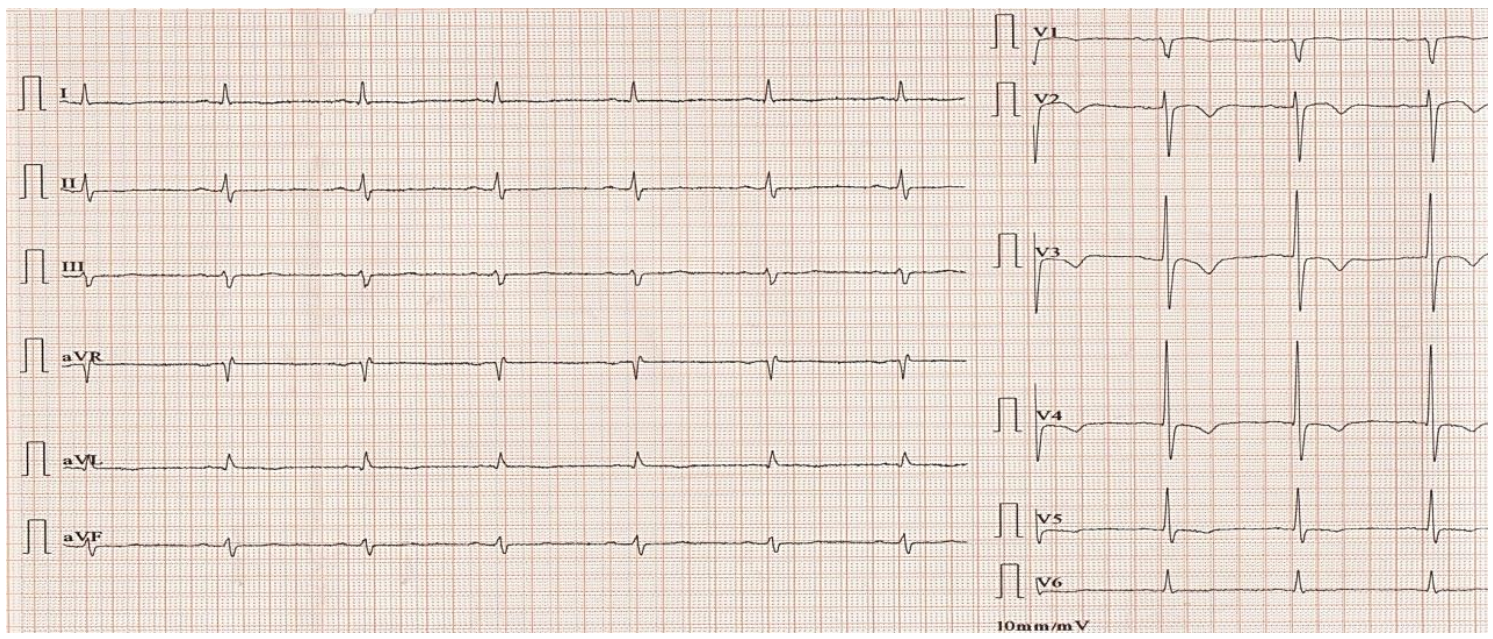

Fig. 1. ECG on admission: normal sinus rhythm and T wave inversions in anterior leads

Echocardiogram showed apical akinesis with reduced left ventricular ejection fraction of $35 \%$, without hemodynamically significant valve disease. Due to high suspicion of acute coronary syndrome she had cardiac catheterization with left ventriculography, which showed mid-anterior and apical akinesia with preserved anterobasal and posterobasal function, with an EF of $35 \%$ (Figure 2). Emergent catheterization revealed no source of cardiac ischemia (Figure 3).
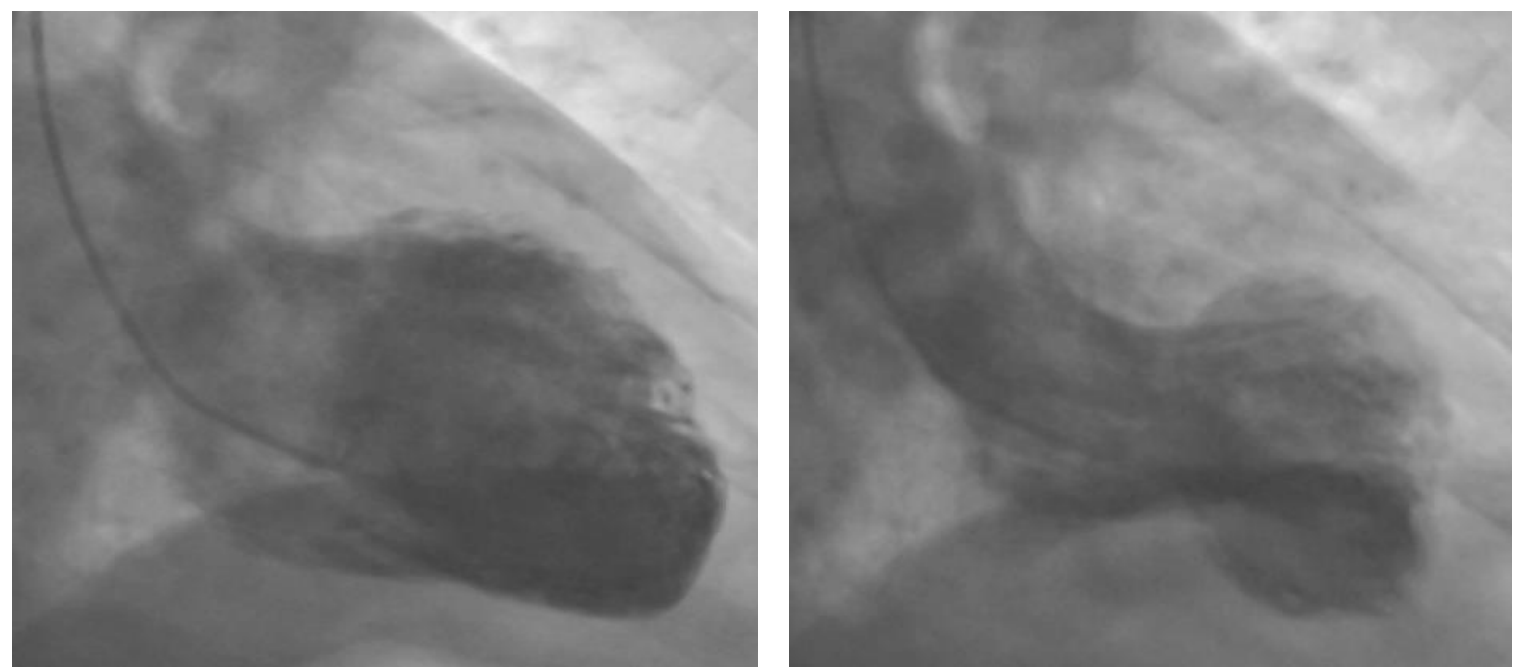

Fig. 2. Left ventriculography with mid-anterior and apical akinesia (left), left ventricular apical ballooning (right) 

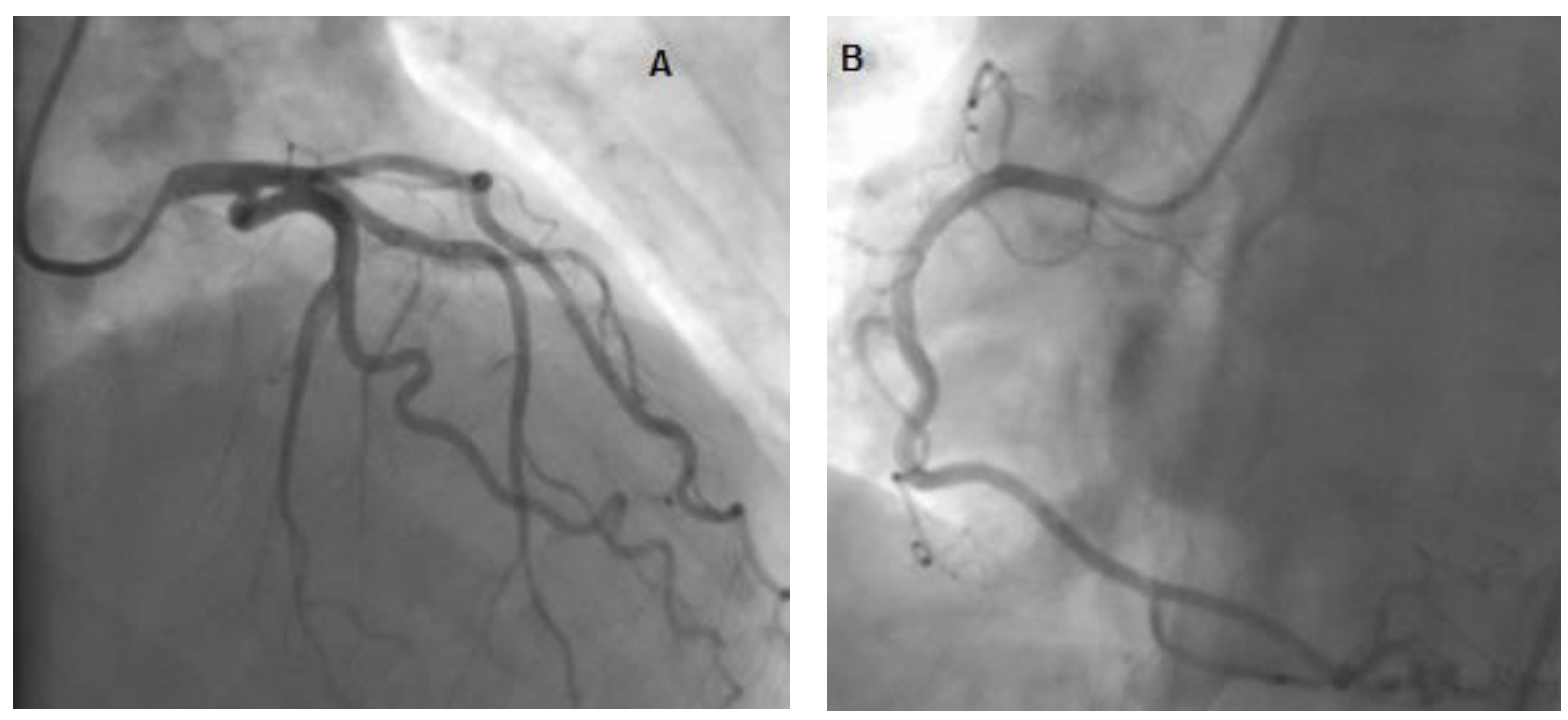

Fig. 3. Coronary angiography with left $(A)$ and right $(B)$ normal coronary artery

Later we resumed the case history in order to find the factor responsible for the occurrence of these symptoms and the only one was the acute pain in the left lower limb in the context of a gonarthrosis.

The patient was hemodynamically stable during the hospitalization under antiagregant therapy, statins and classic medical treatment of left heart failure.

After one month the patient came for cardiology control which revealed the persistence of the ECG changes (Figure 4) but normalization of the left ventricular systolic function.

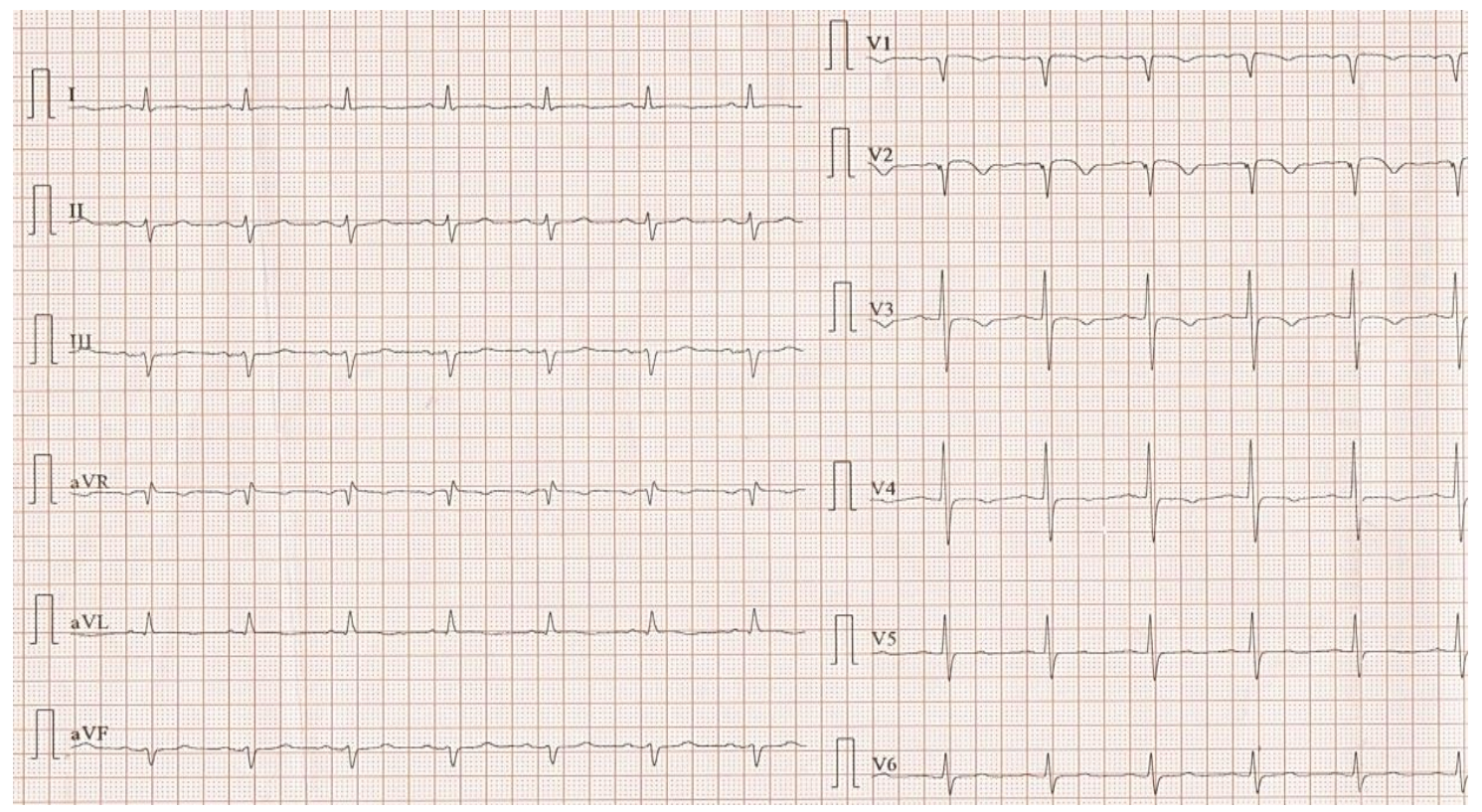

Fig. 4. ECG after one month: normal sinus rhythm with persistent $T$ wave inversions in anterior lead

\section{Discussions}

Our study shows a particular case of Takotsubo cardiomyopathy, due to the important comorbidities of the patient that limits the therapeutic strategies, and also the rare factor that preceded the development of symptoms.

Takotsubo cardiomyopathy is a rare form of reversible heart failure that has been 
reported more recently, in Europe and the United States; it was first reported in Japan. The prevalence of the syndrome is unknown, although various researchers have reported that $1.7-2.2 \%$ of patients with suspected acute coronary syndrome at their institutions are diagnosed with Takotsubo cardiomyopathy [5]. Wellens'sign has also been seen as a rare presentation of Takotsubo cardiomyopathy. It is an electrocardiographic manifestation of a critical proximal left anterior descending coronary artery stenosis in patients with unstable angina and is characterized by symmetrical, often deep $T$ wave inversions in the anterior precordial leads, little or no cardiac marker elevation, discrete or no ST segment elevation and no loss of precordial $R$ waves [6]. An emotional or physical stressor frequently precedes the development of symptoms ( $26.8 \%$ and $37.8 \%$, respectively) [6, 7]. These stressors have included: learning of the death of a loved one, bad financial news, significant arguments, legal problems, car accidents, natural disasters, exacerbation of chronic medical illness, new significant medical diagnosis, surgery and other medical procedures, staying in an intensive care unit, and use of or withdrawal from illicit or narcotic drugs $[8,9]$.

Diagnostic criteria for Takotsubo cardiomyopathy have not been established, but both American and Japanese researchers have proposed similar criteria for the diagnosis. The fourth important findings for the diagnostic of Takotsubo Cardiomyopathy are: (1) transient akinesis or dyskinesis of the apical and midventricular segments in association with regional wall motion abnormalities that extend beyond the distribution of a single epicardial vessel; (2) immediate ST segment elevation on ECG followed by $T$ waves that become progressively more negative and a prolongation of the QT interval; (3) only a modest elevation of cardiac markers; and (4) absence on angiography of obstructive coronary artery disease or evidence of acute plaque rupture. There are also forth similar exclusion criteria: (1) recent significant head trauma or intracranial bleeding, (2) cerebrovascular disease, pheochromocytoma, and (4) myocarditis [10].
These criteria have not been validated, but can be used as guidelines that, when present, should raise suspicion of TCM. Although coronary angiography is the best single tool to diagnose the stress-induced cardiomyopathy, echocardiography is the most important imaging modality to distinguish this syndrome from acute myocardial infarction. Abnormalities in the left ventricular wall motion show a regional or global pattern with a relative hypercontractile base in most cases. There is also a variant of inverted takotsubo pattern (mid-ventricular ballooning with sparing of the basal and apical segments). The dysfunction and regional wall motion abnormalities of the right ventricle are found in $30 \%$ of patients who tend to develop congestive heart failure and who have a poor outcome [11-13].

Several emergency department circumstances may lead to TCM presentation. The syndrome may occur in younger patients without cardiac risk factors or as a consequence of natural disasters. Some anxious or emotionally distraught patients with chest pain may be more complicated than simple anxiety disorder, and may develop arrhythmias or shock [14]. In our case, the evolution of the patient during the hospitalization was stable, without arrhythmias or other complications.

Treatment for Takotsubo cardiomyopathy is similar with the one from an acute coronary syndrome and consists of supportive care of the patient during the period of reversible heart failure with hemodynamic support and appropriate treatment of complications, most commonly left ventricular dysfunction and arrhythmias. The prognosis of patients with TCM is good, with low in-hospital mortality and no reported increased risk of cardiac or other long-term morbidity or mortality $[15,16]$.

The particularity of our case is the fortuitous diagnosis of Takotsubo cardiomiopathy in the context of an acute pain in the left lower limb which we consider the trigger factor, taking into the count the absence of the classical stressors that are involved in the appearance of the diasease. We don't consider that the breast cancer diagnosed and treated ten years ago can be considered the trigger factor for Takotsubo 
cardiomiopathy because the patient has completely recovered after the surgery.

Another particularity of our case is represented by the significant comorbidities and especially repeated episodes of deep vein thrombosis with therapeutic limitations to anticoagulation and antiplatelet therapy due to side effects. Also, the presence of an acute pain in the left lower limb in our patient may suggest the presence of a new episode of deep vein thrombosis but the clinical exam, laboratory studies and the echocardiography denied this hypothesis.

\section{Conclusions}

TCM is a rare but potentially fatal condition, initially indistinguishable from acute coronary syndrome and the diagnosis should be considered in patients with chest pain and a recent stressful event, especially elderly females. According to the proposed criteria for diagnosis, TCM is characterized by presentation and electrocardiographic changes similar to those caused by myocardial infarction; however, atherosclerotic coronary artery disease is usually absent and the transient left ventricular apical akinesis is

\section{References}

1. Kurisu S, Sato H, Kawagoe $T$, et al. Takotsubo-like left ventricular dysfunction with STsegment elevation: a novel cardiac syndrome mimicking acute myocardial infarction. $A m$ Heart J 2002; 143:448-455.

2. Bybee KA, Prasad A, Barsness GW, et al. Clinical characteristics and thrombolysis in myocardial infarction frame counts in women with transient left ventricular apical ballooning syndrome. Am J Cardiol 2004; 94:343-346.

3. Bybee KA, Murphy J, Prasad A, et al. Acute impairment of regional myocardial glucose uptake in the apical ballooning (Takotsubo) syndrome. J Nucl Cardiol 2006; 13:244-250.

4. Gianni M, Dentali F, Grandi AM, et al. Apical ballooning syndrome or Takotsubo cardiomyopathy: a systematic review. Eur Heart J 2006; 27:1523-1529.

5. Donohue D, Movahed MR. Clinical characteristics, demographics, and prognosis usually beyond the distribution of a single coronary artery. In almost all cases an emotional or physiological stressor is identified. These findings are followed by a prompt resolution of symptoms with complete echocardiographic and clinical recovery of cardiac pump function within 2-3 weeks of presentation.

This case report represents a diagnostic and also a therapeutic challenge because of the fortuitous diagnosis in the context of acute pain in the left lower limb which may also be considered the trigger factor for the Takotsubo cardiomyopathy.

\section{Competing interests}

The authors declare that they have no competing interests.

\section{Authors' contributions}

LA and CAG conceived of the case report and drafted the manuscript. LM and MB performed and interpreted diagnostic studies, provided images for figures, and critically revised the manuscript for important intellectual content. All authors read and approved the final manuscript. of transient left ventricular apical ballooning syndrome. Heart Fail Rev 2005; 10:311-316.

6. Park JH, Kang SJ, Song JK, et al. Left ventricular apical ballooning due to severe physical stress in patients admitted to the medical ICU. Chest 2005; 128:296-302.

7. Arora A, Alfayoumi F, Srinivasan V. Transient left ventricular apical ballooning after cocaine use: is catecholamine cardiotoxicity the pathologic link? Mayo Clin Proc 2006; 81:829832.

8. Inoue $\mathrm{M}$, Shimizu $\mathrm{M}$, Ino $\mathrm{H}$, et al. Differentiation between patients with Takotsubo cardiomyopathy and those with anterior acute myocardial infarction. Circ J 2005; 69:89-94.

9. Bybee KA, Kara T, Prasad A, et al. Systematic review: transient left ventricular apical ballooning: a syndrome that mimics STsegment elevation myocardial infarction. Ann Intern Med 2004; 141:858-865. 
10. Kawai S, Kitabatake A, Tomoike $\mathrm{H}$, et al. Guidelines for diagnosis of Takotsubo (ampulla) cardiomyopathy. Circ J 2007; 71:990992.

11. Pellicia F, Kaski JC, Crea F, Camici PG. Pathophysiology of Takotsubo Syndrome. Circulation 2017; 135 (24):2426-2441.

12. Galiuto L, Badano L, Fox $K$, Sicari $R$, Zamorano JL. The EAE Textbook of echocardiography. Oxford University Press 2011.

13. Georgescu Arsenescu C, Floria M. Ghid de ecocardiografie transtoracica, volumul I. Editura Grigore T. Popa 2015. CNCSIS 229. ISBN 978-606-544-295-5.
14. Kurisu S, Inoue I, Kawagoe $\mathrm{T}$, et al. Time course of electrocardiographic changes in patients with Tako-tsubo syndromecomparison with acute myocardial infarction with minimal enzymatic release. Circ $J$ 2004; 68:77-81.

15. Giordan M, Rigatelli G, Cardaioli $P$, et al. Angiographic long-term follow-up of primary apical ballooning of the left ventricle. Int $J$ Card Imag 2006; 22:349-352.

16. Fazio G, Pizzuto C, Barbaro G, et al. Chronic pharmacological treatment in Takotsubo cardiomyopathy. Int J Cardiol 2008; 127:121123. 